\title{
Componentwise block partitioning: a new strategy to solve stiff ordinary differential equations
}

\begin{abstract}
Componentwise Block Partitioning is a new strategy to solve stiff ODEs, based on Block Backward Differentiation Formulas (BBDFs), and block of Adam type formulas. In this partitioning technique, the ODEs system is initially solved by Adam formulas until the equation that cause instability and stiffness is identified. Then, the equations that caused instability are placed into stiff subsystem and solved using BBDF. Numerical comparisons with code in the literature such as ode15s show the efficiency of the proposed partitioning technique.
\end{abstract}

Keyword: Adam; Block backward differentiation; Componentwise block partitioning; Stiff 